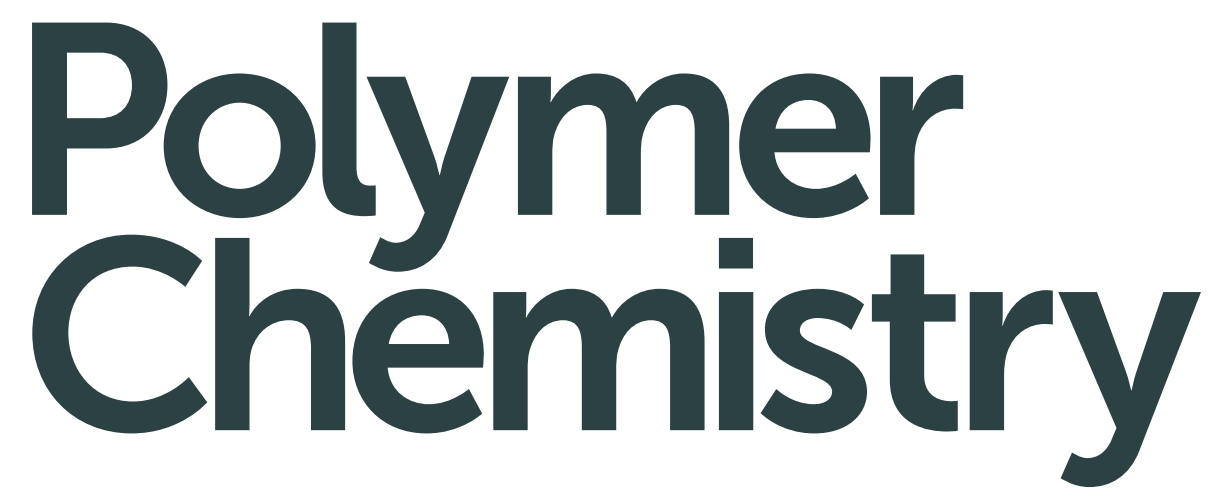

www.rsc.org/polymers

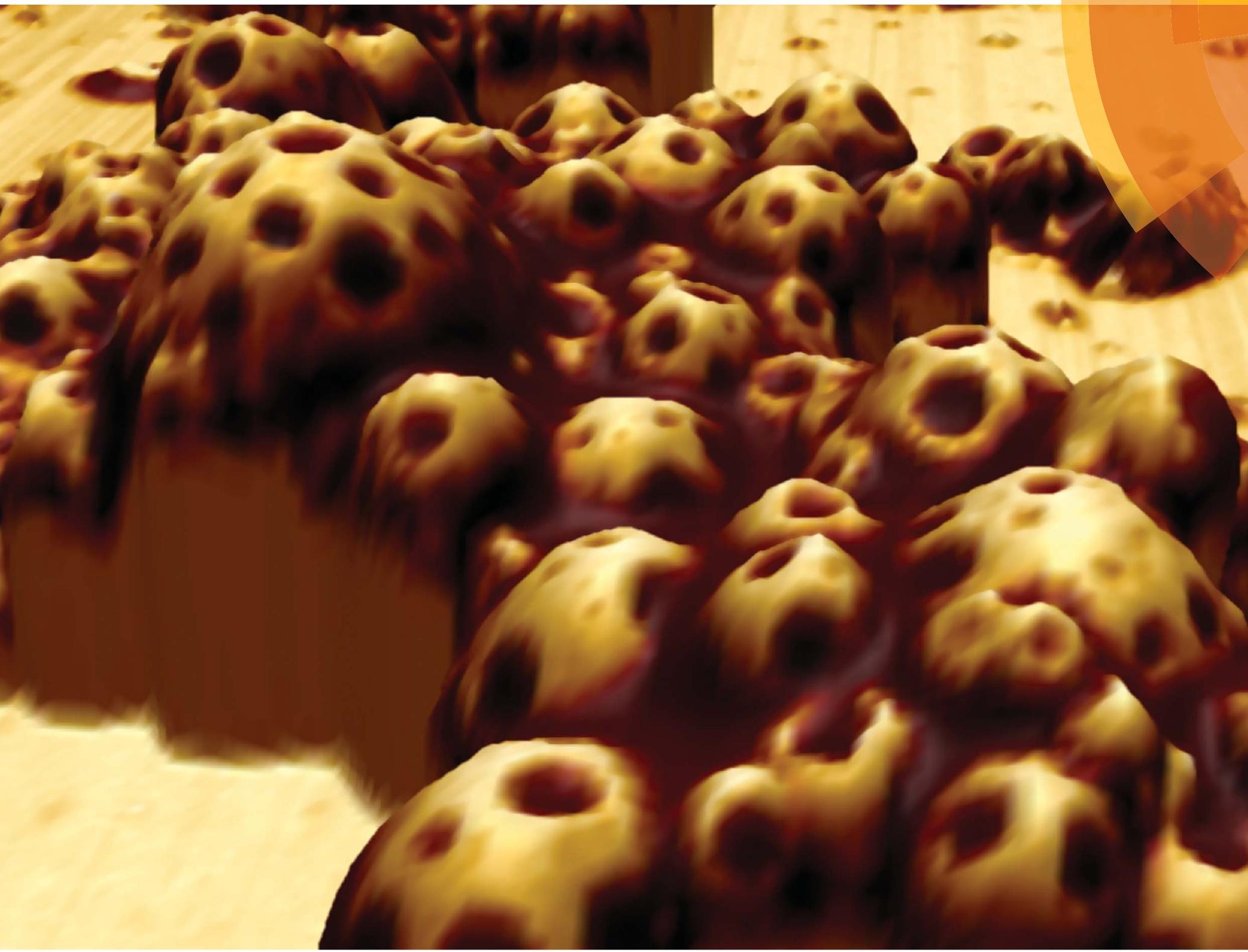

ISSN 1759-9954

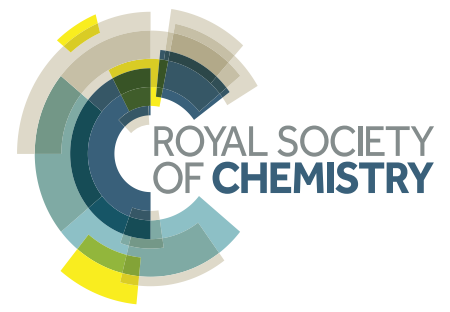




\title{
Polymer patchy colloids with sticky patches $\uparrow$
}

Cite this: Polym. Chem., 2014, 5, 365

Received 13th August 2013

Accepted 11th September 2013

DOI: $10.1039 /$ c3py01096a

www.rsc.org/polymers

\author{
Yi Zhao, Rüdiger Berger, Katharina Landfester and Daniel Crespy*
}

Patchy particles (PPs) are considered as interesting building blocks for the fabrication of novel structures with enhanced complexity and functionality. However, their development is primarily limited by the fact that there is no reliable method to prepare PPs on a large scale. Herein, a one-pot strategy to prepare PPs relying on polymerization-induced phase separation in monomer-embedded polymer nanoparticles is demonstrated. The surface is found to be composed of sticky patches embedded in a hard matrix by adhesion and force-distance measurements performed by atomic force microscopy. The patch sizes could be easily tuned by controlling the monomer conversion or varying the composition between the polymer and the monomer. This study presents the possibility to develop a simple, low-cost and scalable method for preparing large quantities of PPs from homopolymers. It may also pave the way to new PPs for functional materials and devices.

\section{Introduction}

Patchy particles (PPs) are colloidal particles with a chemically or physically patterned surface. ${ }^{\mathbf{1 - 3}}$ Janus particles can be considered as the simplest PPs with only one patch. ${ }^{4-6}$ The patchy domains may provide specific and directional interactions with other particles or surfaces and therefore PPs can self- and direct-assemble into novel suprastructures which can find applications in the delivery of drugs or other chemicals, ${ }^{7-9}$ electronic devices, ${ }^{10,11}$ photonic crystals, ${ }^{12,13}$ and sensors. ${ }^{14-16}$ Currently, the preparation of PPs mainly focuses on templating or chemical patterning, ${ }^{17-22}$ glancing angle deposition, ${ }^{23-25}$ particle lithography, ${ }^{26-29}$ capillary fluid flow, ${ }^{30-32}$ and selfassembly of pre-synthesized block copolymers., ${ }^{9,33-37}$ These approaches, however, are difficult to scale up because of demanding synthetic routes or owing to intrinsic limitations related to the preparation process, and therefore hamper the study and development of PPs. Recently, silica particles were decorated with silver or gold patches by heterogeneous nucleation of silver nitrate or a dark-aged solution of potassium carbonate and chloroauric acid. ${ }^{38-40}$ The method is scalable but limited to inorganic patches. Okubo et al. prepared polymer particles with dents on the surface by seeded emulsion polymerization $^{\mathbf{4 1 - 4 3}}$ and seeded dispersion polymerization. ${ }^{\mathbf{4 4 5}}$ However, an effort is required to synthesize seed particles first. Seeded dispersion or emulsion polymerization was also applied to synthesize non-spherical particles due to a polymerizationinduced phase separation between the original polymer and the newly formed polymer, ${ }^{\mathbf{4 6}-48}$ but they are usually limited to

Max Planck Institute for Polymer Research, Ackermannweg 10, 55128 Mainz, Germany.E-mail: crespy@mpip-mainz.mpg.de

$\uparrow$ Electronic supplementary information (ESI) available: Detailed characterization by SEM, TEM, AFM, DSC and ${ }^{1}$ H NMR. See DOI: 10.1039/c3py01096a the preparation of particles with only one patch and having large size, e.g. several micrometers. Miniemulsion polymerization is a powerful method to develop nanoparticles with different compositions and architectures. ${ }^{49}$ We previously showed that patchy nanoparticles and nanocapsules, based on poly(methyl methacrylate-block-styrene $)^{36}$ or poly(methyl methacrylate-block-vinylferrocene), ${ }^{9}$ could be prepared by a combination of miniemulsion and solvent evaporation techniques. The preparation of polymer PPs in large quantities and with multiple patches by a facile and seed-free method without employing block copolymers still remains a great challenge.

In this study, a monomer and an initiator were firstly embedded in nanoparticles by using the aforementioned miniemulsion-solvent evaporation technique. It was demonstrated that this technique could be executed without significant occurrence of coalescence ${ }^{\mathbf{5 0 , 5 1}}$ and was found to be suitable for the encapsulation of various liquid chemicals. ${ }^{52-54}$ PPs were then easily obtained by polymerizing the monomer because polymerization could induce phase separation between the newly formed polymer and the original polymer and induced the formation of sticky patches. We describe here a simple and scalable strategy to prepare PPs with tunable patch sizes without block copolymers. Grams of PPs could be easily prepared based on bench chemistry in one day.

\section{Experimental section}

\section{Materials}

Poly(vinyl formal) (PVF, $M_{\mathrm{w}} \sim 10000 \mathrm{~g} \cdot \mathrm{mol}^{-1}$, Aldrich), sodium dodecyl sulfate (SDS, 99\%, Aldrich), deuterium oxide $\left(\mathrm{D}_{2} \mathrm{O}, 99.9 \%\right.$, Aldrich), dimethyl sulfoxide- $d_{6}$ (DMSO- $d_{6}, 99.9 \%$, Aldrich), hexadecane (HD, 99\%, Merck), azobisisobutyronitrile (AIBN, 98\%, Aldrich), dichloromethane (DCM, 99\%, VWR), chloroform $\left(\mathrm{CHCl}_{3}\right.$, 99\%, VWR), 2,2-dimethoxy-2-phenylacetophenone (DMPA, 99\%, 
Aldrich), potassium persulfate (KPS, 99.9\%, Aldrich), 1,4-divinylbenzene (DVB, 99\%, Aldrich) and argon (Ar, 99.99\%, Westfalen AG) were used as received. Dodecyl methacrylate (DMA, 96\%, Aldrich) was passed through an aluminum oxide (basic) column before use. The chemical structures of the monomers, initiators and polymers are shown in Fig. S1. $\dagger$

\section{Preparation of PVF-DMA nanoparticles}

Solvent evaporation and miniemulsion techniques were applied to prepare PVF-DMA nanoparticles. Known amounts of PVF, DMA and initiators (see Table 1 for the details) were dissolved in $5 \mathrm{~mL} \mathrm{CHCl}_{3}$, and stirred at $600 \mathrm{rpm}$ for $20 \mathrm{~min}$. Then a solution of SDS in $\mathrm{D}_{2} \mathrm{O}$ solution $\left(10 \mathrm{~mL} \times 1.0 \mathrm{mg} \mathrm{mL} \mathrm{mL}^{-1}\right)$ was added, followed by stirring at $1100 \mathrm{rpm}$ for $1 \mathrm{~h}$. The obtained emulsion was then treated with a sonifier (Branson W450D Digital, 1/2 inch tip, 90\% amplitude) for $2 \mathrm{~min}(30 \mathrm{~s}$ pulse and $15 \mathrm{~s}$ pause, $4 \times$ ) in an ice-bath. The obtained miniemulsion was stirred at $40{ }^{\circ} \mathrm{C}$ overnight to evaporate $\mathrm{CHCl}_{3}$. For evaluating the evaporation speed of $\mathrm{CHCl}_{3}$, aliquots of the dispersion were taken each hour during the first experiment (entry 1 of Table 1 ) for ${ }^{1} \mathrm{H}$ NMR measurements. For PVF-HD nanoparticles (entry 3 in Table 1), the same preparative procedure was adapted.

\section{Generation of patchy nanoparticles}

The dispersion was bubbled with Ar for 10 min before heating at $72{ }^{\circ} \mathrm{C}$. To follow the conversion of DMA, an aliquot of the dispersion was taken every $15 \mathrm{~min}$ for ${ }^{1} \mathrm{H}$ NMR measurement. For all the samples with AIBN as the initiator, the polymerization was completed in 30 min (see Fig. S2 $\dagger$ ) with DMA conversions of $\sim 90$ to $97 \%$. The evolution of the colloidal morphologies depending on the DMA conversion measured by ${ }^{1} \mathrm{H}$ NMR (entry 7 of Table 1 ) was carried out by taking aliquots of the dispersion every $5 \mathrm{~min}$. At the same time, samples were taken for SEM measurements. When KPS was used as the initiator (entry 2 of Table 1), KPS was added before bubbling Ar. In the case of DMPA as the photoinitiator, the dispersion was transferred into a quartz cuvette and heated at $72{ }^{\circ} \mathrm{C}$ for $20 \mathrm{~min}$ under UV irradiation ( $\mathrm{Hg}$ lamp, UV-Consulting Peschl TQ 150,

Table 1 Composition and hydrodynamic diameters of the PPs

\begin{tabular}{llrll}
\hline Entry & PVF $[\mathrm{mg}]$ & DMA $[\mathrm{mg}]$ & DVB $[\mathrm{mg}]$ & $D_{\mathrm{h}}[\mathrm{nm}]^{a}$ \\
\hline 1 & 250 & 250 & 0 & $240 \pm 70$ \\
$2^{b}$ & 400 & 100 & 0 & $230 \pm 90$ \\
$3^{c}$ & 400 & 0 & 0 & $250 \pm 70$ \\
$4^{d}$ & 400 & 100 & 0 & $280 \pm 90$ \\
5 & 450 & 50 & 0 & $225 \pm 50$ \\
6 & 400 & 100 & 0 & $300 \pm 105$ \\
7 & 300 & 200 & 0 & $250 \pm 80$ \\
8 & 200 & 300 & 0 & $280 \pm 85$ \\
9 & 100 & 400 & 30 & $350 \pm 140$ \\
10 & 300 & 170 & 20 & $260 \pm 70$ \\
11 & 300 & 180 & 10 & $230 \pm 80$ \\
12 & 300 & 190 & & $275 \pm 80$
\end{tabular}

${ }^{a}$ Measured by DLS. ${ }^{b}$ KPS as an initiator. ${ }^{c}$ DMA was replaced by $100 \mathrm{mg}$ hexadecane. ${ }^{d}$ 2,2-dimethoxy-2-phenylacetophenone (DMPA) as an initiator. cooling with water, $150 \mathrm{~W}$ ). The distance between the lamp and cuvette was $10 \mathrm{~cm}$. A control test was carried out at room temperature with the same procedure.

\section{Characterization of the polymers and particles}

The hydrodynamic diameters of the particles in diluted dispersions were measured with a dynamic light scattering machine (NICOMP 380, Santa Barbara) at a fixed angle of $90^{\circ}$ and a laser diode running at $635 \mathrm{~nm} .{ }^{1} \mathrm{H}$ NMR measurements were carried out on a Bruker AVANCE $300 \mathrm{MHz}$ console at room temperature using DMSO- $d_{6}$ as the solvent. Samples were prepared by diluting $25 \mu \mathrm{L}$ of the dispersion in $0.55 \mathrm{~mL}$ DMSO- $d_{6}$. The morphology of particles was observed with a Gemini 1530 (Carl Zeiss AG, Oberkochem, Germany) scanning electron microscope (SEM) running at $0.3 \mathrm{kV}$. Samples for SEM measurements were prepared by diluting $25 \mu \mathrm{L}$ of dispersion in $3 \mathrm{~mL}$ distilled water, casting one drop of the diluted dispersion on a $0.5 \times 0.5 \mathrm{~cm}^{2}$ silica wafer, and left to dry at room temperature. The morphology of particles was further studied by using a transmission electron microscope (TEM) (Zeiss EM912) with an accelerating voltage of $120 \mathrm{kV}$. Samples were prepared by casting one drop of diluted dispersion on a carbon-layer-coated copper grid (300 square mesh) and dried at room temperature. For ultrathin crosssectional TEM measurement, the PPs were first stained with osmium tetroxide $\left(\mathrm{OsO}_{4}\right)$ for $45 \mathrm{~min}$ in dispersion. After centrifugation of the dispersions, the PPs were washed with water several times. Water was gradually replaced by a graded series of ethanol ( $\sim 1$ day), and then ethanol was gradually replaced by a graded series of EPON ( $\sim 1$ day) at room temperature. Afterwards, the PPs were embedded in EPON and cured at $60{ }^{\circ} \mathrm{C}$ for 3 days. Ultrathin cross-sectional samples $(\sim 60 \mathrm{~nm})$ were prepared with a Leica microtome and studied on a Philips Tecnai F20 electron microscope at an operating voltage of $200 \mathrm{kV}$.

Atomic force microscope (AFM) images were recorded with a commercial Bruker Dimension 3100 (NanoScope IIIa controller) setup in tapping mode. The mechanical measurements (topography, adhesion and slope) were performed with a NanoWizard 3 using the quantitative imaging mode. For this mode we used Olympus probes (OMCL AC240TS) that were calibrated first on a hard Silicon wafer. Then the spring constant was determined by thermal tuning. The topography was obtained at a setpoint of $500 \mathrm{pN}$. All images in QI-mode were recorded with $256 \times 256$ pixels. Samples were prepared by casting one drop of diluted dispersion on a silica wafer and dried at room temperature.

The glass transition temperatures $\left(T_{\mathrm{g}}\right)$ for the PVF and the PVF-DMA mixture were measured on a differential scanning calorimetric (DSC) machine (Mettler Toledo DSC 823) operating at a heating rate of $10{ }^{\circ} \mathrm{C} \mathrm{min}^{-1}$ under $\mathrm{N}_{2}$. The PVF-DMA blend was prepared by mixing $400 \mathrm{mg}$ PVF and $100 \mathrm{mg}$ DMA in $5 \mathrm{~mL}$ dichloromethane (DCM), and then evaporating DCM at room temperature. A powder of PPs was obtained by freeze-drying, and then annealed at $120{ }^{\circ} \mathrm{C}$ for $24 \mathrm{~h}$. A sample without annealing was also prepared as control experiment. The $T_{\mathrm{g}}$ 
values were evaluated as the midpoint of the change in heat capacity.

\section{Results and discussion}

A monomer (dodecyl methacrylate, DMA) and an initiator (AIBN, azobisisobutyronitrile) for radical polymerization were first embedded in polymer (PVF, poly(vinyl formal)) nanoparticles by a miniemulsion-solvent evaporation process. PVF and DMA were chosen as the polymer/monomer pair, because PVF has a glass transition temperature $\left(T_{\mathrm{g}}\right)$ of $\sim 105{ }^{\circ} \mathrm{C},{ }^{55}$ which is beneficial to lock the formed structures at room temperature while PDMA has a $T_{\mathrm{g}}$ of $\sim-48{ }^{\circ} \mathrm{C},{ }^{56}$ which is helpful to increase the mobility of PDMA chains during the polymerization and therefore accelerate the phase separation. Unless otherwise stated, chloroform $\left(\mathrm{CHCl}_{3}\right)$ was applied as a good solvent to dissolve PVF and DMA and sodium dodecyl sulfate (SDS) was used to stabilize the particles. ${ }^{1} \mathrm{H}$ NMR spectroscopy was carried out to follow the evaporation of $\mathrm{CHCl}_{3}$ from the droplets/ nanoparticles. It was found that $10 \mathrm{~h}$ were necessary under the experimental conditions to completely remove $\mathrm{CHCl}_{3}$ from the dispersions (Fig. 1a). In our study, the evaporation time was fixed to $15 \mathrm{~h}$ to ensure complete removal of $\mathrm{CHCl}_{3}$ before polymerization. Indeed, $\mathrm{CHCl}_{3}$ is a good solvent for both $\mathrm{PVF}$ and PDMA and it is therefore necessary to remove it in order to allow phase separation in the droplets. The polymerization of DMA was followed by ${ }^{1} \mathrm{H}$ NMR. The disappearance of peaks at 5.66 and $6.0 \mathrm{ppm}$ (the protons connect to the carbon carbon double bond, shown by arrows in Fig. 1b) proved that DMA was fully polymerized.

A representative dispersion of PPs was prepared by using a weight ratio PVF : DMA of 1 : 1 (entry 1 in Table 1). After evaporation of $\mathrm{CHCl}_{3}$ but before polymerization of DMA, the surface of the nanoparticles was found to have bucket-like morphologies with smooth surfaces (Fig. 2a). These bucket-like morphologies are due to the evaporation of DMA under high vacuum in the chamber for scanning electronic microscopy. After polymerization of DMA, many patches looking like depressions in SEM appeared on the particle surface as shown in Fig. 2b. These patches are attributed to dimples, which were caused by shrinkage of the PDMA domains. Indeed, the polymerization induces a volume reduction due to an increase in

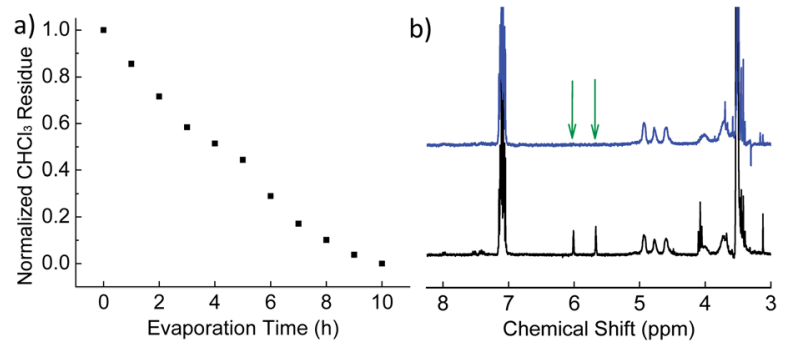

Fig. 1 (a) Normalized $\mathrm{CHCl}_{3}$ residue as a function of evaporation time (entry 1 in Table 1); (b) ${ }^{1} \mathrm{H}$ NMR spectra of the dispersion before (black) and after (blue) polymerization at room temperature (entry 4 in Table 1); xylene (7.02-7.16 ppm) was used as an external standard.

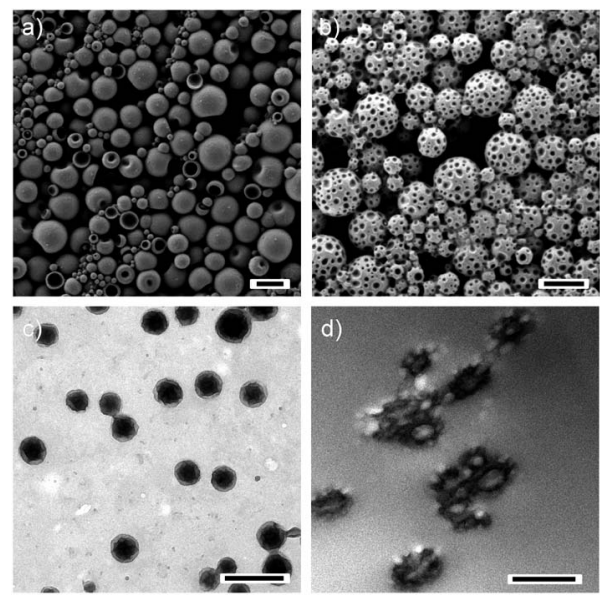

Fig. 2 Representative SEM micrographs of the nanoparticles (entry 1 of Table 1) (a) before and (b) after polymerization of DMA. (c) TEM and (d) cross-sectional TEM micrographs of the same PPs. The scale bars represent $500 \mathrm{~nm}$.

density from $0.868 \mathrm{~g} \mathrm{~cm}^{-3}$ (DMA) ${ }^{57}$ to $0.929 \mathrm{~g} \mathrm{~cm}^{-3}$ (PDMA). ${ }^{58}$ The dimples on the PP surface were also detected by TEM microscopy (Fig. 2c). To better visualize the phase separation between PVF and PDMA in the whole particles, cross-sectional TEM micrographs were taken for PPs after staining with osmium tetroxide $\left(\mathrm{OsO}_{4}\right)$. It is known that $\mathrm{OsO}_{4}$ can be used to selectively stain PVF under our experimental conditions. ${ }^{59}$ The thickness of the sample was $\sim 60 \mathrm{~nm}$, less than the diameter of particles of $\sim 250 \mathrm{~nm}$. As shown in Fig. $2 \mathrm{~d}$ and Fig. S3, $\dagger$ coreshell structures could be clearly identified. The brighter core was created because some of the PDMA diffused out of the particles during the sample preparation whereas the dark domains belong to the stained PVF.

Although the cross-sectional TEM micrograph in Fig. 2d shows that there are two different chemical domains on the particle surface, the sizes, shapes and depths of the domains cannot be measured accurately because of the fact that the particles are deformed during the cross-sectioning. Atomic force microscopy (AFM) was carried out on the particles to exclude any effect of the vacuum required for electron microscopy on the morphology of the colloids (Fig. 3). The AFM phase contrast images revealed two distinct phases on the PP surface. The diameter of the patches of the dark phase contrast ranged from 10 to $60 \mathrm{~nm}$ (Fig. 3b). These patches were also visible in AFM topography as dimples in the PP (Fig. 3a). Again this can be explained by the smaller height of the patches induced by the contraction of the PDMA during the polymerization. Thus the dark phase contrast areas should correspond to PDMA. A more direct proof that these patches correspond to PDMA is to measure the surface elasticity and the tip sample adhesion forces using force distance curves. Such a study was performed on several areas and the data recorded on a single PP are exemplarily presented in Fig. 3c-e. The topography image (Fig. 3c) revealed no difference compared to standard AFM imaging. The surface elasticity revealed a reduced slope in the PDMA-rich domains compared to the surrounding material (Fig. 3d) while the adhesion in these areas was measured to be 

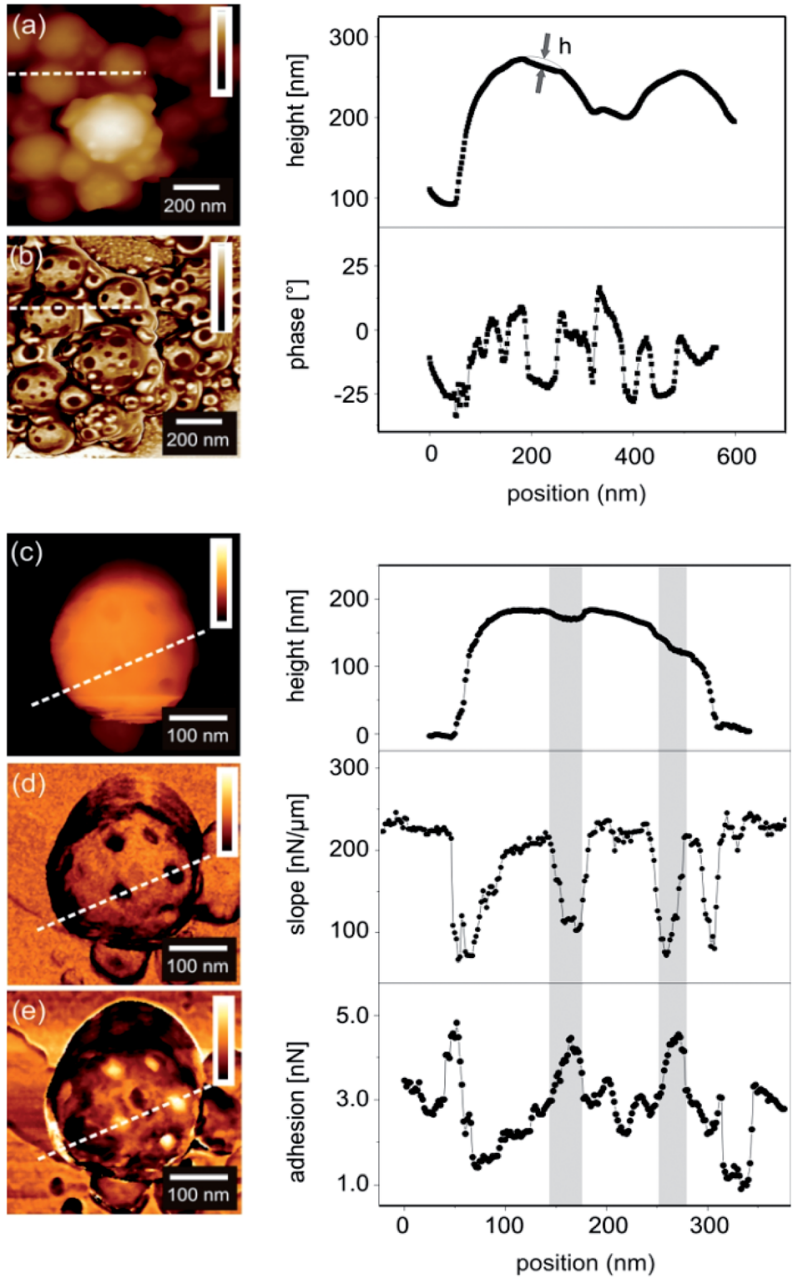

Fig. 3 AFM height (a) and phase (b) images of PPs (entry 1 of Table 1), 1 $\mu \mathrm{m} \times 1 \mu \mathrm{m}$; the right curves show a topography profile. The depth of the dimple $h$ was calculated by subtracting the profile from a spherical profile. The average depth of the patch estimated by AFM is between 5 and $20 \mathrm{~nm}$. (c-e) Quantitative AFM images of a single patchy particle. The areas in the graphs marked in grey represent the locations of the patches. In (e), the background variation in the adhesion forces was attributed to the presence of remaining surfactant molecules on the particle surface.

increased (Fig. 3e). This way we could clearly show where the low $T_{\mathrm{g}}$ material, i.e. PDMA, is situated in the PPs.

In order to clearly understand if there are other factors influencing the generation of PPs, a series experiments were carried out as follows. The miscibility between PVF and the monomer DMA was studied by differential scanning calorimetric (DSC) measurements. Fig. 4a shows that the pure PVF has a $T_{\mathrm{g}} \sim 105{ }^{\circ} \mathrm{C}$ (2nd scan, heating curve), whereas the $T_{\mathrm{g}}$ of the PVF : DMA blend of $4: 1$ (wt : wt) was decreased to $\sim 85{ }^{\circ} \mathrm{C}$. This is due to the plasticizing effect that DMA induces for PVF, which means PVF and DMA are partially miscible. Another hint for the presence of DMA in the PVF is given by the fact that the patchy structure can also be obtained using a water soluble initiator instead of AIBN after the evaporation of $\mathrm{CHCl}_{3}$ (Fig. 4b). The polymerization initiated by potassium persulfate (KPS) yielded PDMA, which means that DMA was partly present on the PVF surface. As AIBN was used as the
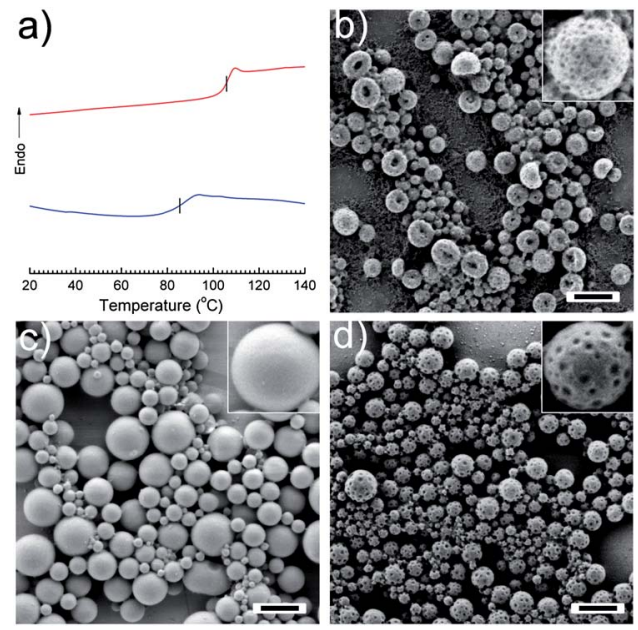

Fig. 4 (a) Differential scanning calorimetric heating curves (2nd scan) for PVF (upper) and PVF-DMA blend (4:1 wt: wt) (lower); SEM micrographs of PVF nanoparticles prepared under different conditions: (b) KPS as an initiator (entry 2 in Table 1); (c) HD as hydrophobic oil (entry 3 in Table 1); (d) DMPA as a photoinitiator (entry 4 in Table 1). The scale bars represent $500 \mathrm{~nm}$.

initiator in most cases, the decomposition of AIBN will generate a considerable amount of nitrogen gas $\left(\mathrm{N}_{2}\right)$. In our study, there is $1.57 \mathrm{~cm}^{3} \mathrm{~N}_{2}$ generated at $72{ }^{\circ} \mathrm{C}$ when AIBN is fully decomposed according to Charles Law, $V_{1} / T_{1}=V_{2} / T_{2}\left(V_{1}\right.$ is the volume of $\mathrm{N}_{2}$ at room temperature $T_{1} ; V_{2}$ is the volume of $\mathrm{N}_{2}$ at reaction temperature $T_{2}$ ). In order to study the possible effect of $\mathrm{N}_{2}$ on the morphology of PPs, DMA was replaced by the chemically inert hexadecane and then the dispersion was treated under the same conditions as for the preparation of PPs. No indentations were detected on the surface of particles (Fig. 4c). The gas generation was therefore not the reason for the formation of the patches. To demonstrate that the polymerization reaction plays the key role in the formation of the PPs, another experiment was conducted to form PPs with the photoinitiator DMPA, which does not release gas during polymerization. As shown in Fig. 4d, dimples were present on the nanoparticle surface when DMA was polymerized at $72{ }^{\circ} \mathrm{C}$. However, no patches could be obtained (see Fig. S4 $\dagger$ ) when DMA was polymerized at room temperature although the conversion of monomer to polymer was $\sim 100 \%$. These results further justify our conclusion that polymerization-induced phase separation is the dominant driving force in forming patches in such PPs. Temperature also plays an important role to allow and accelerate the phase separation during polymerization.

The temporal evolution of the nanoparticle morphology as a function of the conversion $\left(C_{\mathrm{m}}\right)$ was followed by SEM (Fig. 5). Aliquots of the dispersion were taken at different times for ${ }^{1} \mathrm{H}$ NMR measurements to determine $C_{\mathrm{m}}$. Phase separation already occurred at a very early stage of polymerization $\left(C_{\mathrm{m}}=6 \%\right.$, Fig. 5b) according to a nucleation-growth mechanism. Some tiny dimples appeared on the particle surface. When $C_{\mathrm{m}}<58 \%$ (Fig. 5a-d), the sizes of the patches increased with increasing $C_{\mathrm{m}}$, e.g. $\sim 30 \mathrm{~nm}$ with $C_{\mathrm{m}}=30 \%$ and $\sim 45 \mathrm{~nm}$ with $C_{\mathrm{m}}=58 \%$. When $C_{\mathrm{m}}>58 \%$ (Fig. $5 \mathrm{e}-\mathrm{h}$ ), the size of the patches was almost constant even when $C_{\mathrm{m}}$ was increased above $90 \%$. This is 


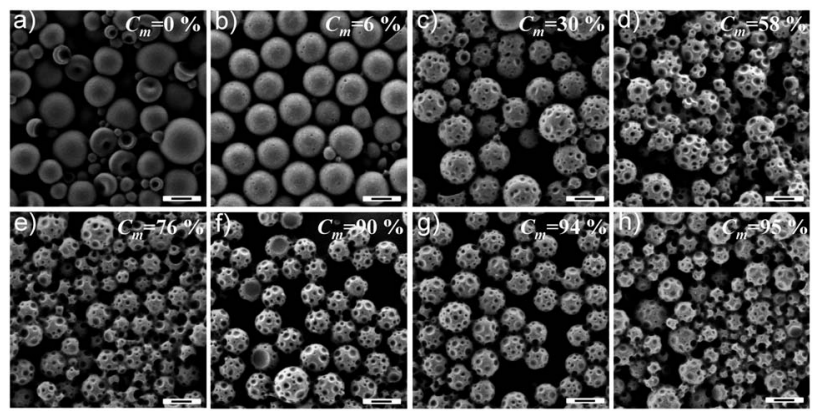

Fig. 5 SEM micrographs show the morphological evolution of PVF nanoparticles (entry 7 in Table 1) with increased conversion of DMA to polymer. The scale bars represent $200 \mathrm{~nm}$.

possibly due to the enhanced viscosity of the system when $C_{\mathrm{m}}$ is high, which slows down the mobility of the polymer chains. Moreover, the aforementioned plasticizing effect of DMA on PVF becomes less pronounced with DMA conversion, leading to a locking of the nanoparticle structure. This means that the size of the patches of the PPs can be tuned by controlling the monomer conversion, especially at the early stage of the polymerization. However, the consequence is that some amount of the monomer can be present in the final PPs.

Therefore we searched for another method to control the size of the patches while minimizing the amount of the non-reacted monomer in the PPs. This can be achieved by precisely adjusting the composition of the PPs (entries 5-9 of Table 1). For concentrations of DMA $<50 \%$ (Fig. S5a-c $\dagger$ and $2 \mathrm{a}$ ), the particles possess spherical morphologies, i.e., the DMA is embedded in the PVF nanocapsules. ${ }^{53}$ Above $50 \%$ of DMA, the DMA cannot be fully encapsulated by PVF and bowl-shaped structures were identified (Fig. S5d $\dagger$ ). As shown in Fig. 6a, the average patche size (calculated on 100 patches) was $\sim 17 \mathrm{~nm}$ when the concentration of the monomer was $10 \%$. Increasing the amount

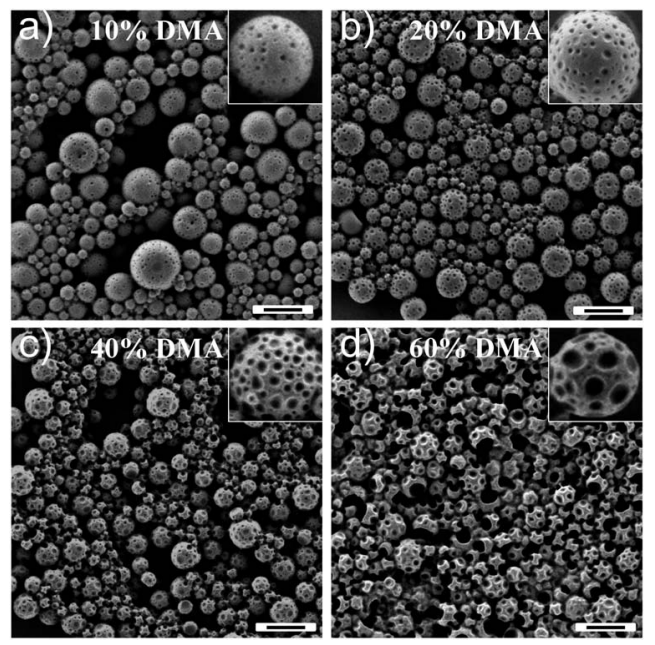

Fig. 6 SEM micrographs of PPs reveal that the patch size could be adjusted by varying the compositions of DMA and for $C_{m}>95 \%$. The scale bars represent $500 \mathrm{~nm}$. The inset particles have a diameter of $\sim 300 \mathrm{~nm}$. of DMA in the nanoparticles to $20 \%$ and $40 \%$ yielded a patch size of $\sim 25 \mathrm{~nm}$ and $\sim 42 \mathrm{~nm}$, respectively (Fig. 6b-c). A further rise in the concentration of DMA $(60 \%)$ leads also to a larger patch size (Fig. 6d), but causes the formation of acorn structures instead of the previously observed patchy nanocaspule structures. Patchy structures were also detected after polymerization when DMA was $80 \%$ but only in the bowl shaped section formed by the PVF (Fig. S6 $\dagger$ ). The size of the patches, therefore, can be controlled to be from $\sim 15 \mathrm{~nm}$ to $\sim 45 \mathrm{~nm}$ by adjusting the composition of the nanoparticles.

The PPs morphologies are in a kinetically trapped but thermodynamically unstable state. Indeed, after annealing the nanoparticles at $T>T_{\mathrm{g}}$, phase separation between the PDMA and the PVF occurred and led to the vanishing of the patchy domains. As shown in Fig. S7, $\uparrow$ heating the PPs (entry 6 of Table 1) at $90{ }^{\circ} \mathrm{C}$ removed the particle surface structures while aggregation of particles was detected (Fig. S7d $\dagger$ ). The thermal properties of the PPs were investigated by DSC to estimate the miscibility of the polymers in the PPs. Two $T_{\mathrm{g}}$ values at $-32{ }^{\circ} \mathrm{C}$ and $77^{\circ} \mathrm{C}$ were detected before annealing, corresponding to the PDMA-rich phase and the PVF-rich phase, respectively (Fig. S8 $\dagger$ ). Compared with the $T_{\mathrm{g}}$ of the individual components $\left(105{ }^{\circ} \mathrm{C}\right.$ for PVF and $-48{ }^{\circ} \mathrm{C}$ for $\mathrm{PDMA}^{56}$ ), these results indicate that the confinement and the kinetically trapped colloidal morphology force a partial mixing of PVF and PDMA. Note that SDS also acts as a plasticizer to decrease the $T_{\mathrm{g}}$ of polymers. ${ }^{\mathbf{6 0 , 6 1}}$ After annealing the freeze dried PPs (as powder) at $120{ }^{\circ} \mathrm{C}$ for $24 \mathrm{~h}$, the measured $T_{\mathrm{g}}$ values of the PPs were found to be shifted oppositely to $-38{ }^{\circ} \mathrm{C}$ and $103{ }^{\circ} \mathrm{C}$, revealing a higher extent of phase separation between both polymers. In order to lock the kinetically trapped patchy morphology, we investigate the possibility to crosslink the PDMA domains. The use of a crosslinker for the formation of PPs is however a double-edge strategy. On the one hand, the colloidal morphology shall be locked. On the other hand, it may hamper the formation of the patchy domains due to the rapid increase of viscosity in the particles with increased monomer conversion. Indeed, PPs prepared with $15 \mathrm{wt} \%$ DVB (1,4-divinylbenzene) as a crosslinker displayed an irregular surface (Fig. S9a†) but no identifiable patchy domains (Fig. S9b $\dagger$ ). Reducing the concentration of the crosslinker to 10 and 5\% (entries 10 and 11 in Table 1) however yielded PPs. Whereas the PPs with a 5\% crosslinker were aggregated upon annealing at $90{ }^{\circ} \mathrm{C}$ for $3 \mathrm{~h}$, the PPs with a $10 \%$
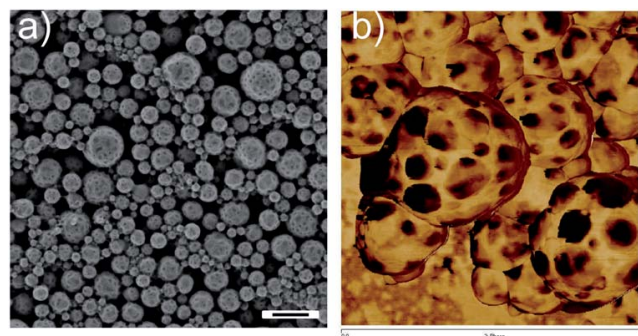

Fig. 7 SEM micrograph (a) and AFM phase contrast image $1 \mu \mathrm{m} \times 1 \mu \mathrm{m}$ (b) of cross-linked PPs (entry 11 in Table 1 ) after treating at $90^{\circ} \mathrm{C}$ for $3 \mathrm{~h}$. The scale bar represents $500 \mathrm{~nm}$. 
crosslinker tolerated the annealing. Although some aggregation was still observed, separated PPs with specific surface domains could be clearly identified (Fig. 7a). Again, the dimples were proved to be of a different composition than the matrix by AFM phase imaging (Fig. 7b). The patchy domains in the PPs could be therefore stabilized by the introduction of an appropriate amount of crosslinker.

\section{Conclusions}

In summary, we demonstrated an easy, low-cost and mild strategy to fabricate patchy particles (PPs) in large quantities without using block copolymers. Polymerization-induced phase separation was found to be the reason for the formation of the PPs. The size of the patches could be easily tuned by controlling the monomer conversion or by changing the composition of the nanoparticles. The atomic force microscopy analysis revealed that the patches were found to be sticky and embedded in a harder polymer matrix. Moreover, the patchy structure could be locked by crosslinking the sticky patches. Our approach could be extended to prepare large libraries of different PPs by choosing other polymer/ monomer pairs and/or by post-functionalizing the patchy area.

\section{Acknowledgements}

The authors acknowledge Haixin Zhou and Petra Räder for DSC measurements, and Uwe Rietzler for AFM imaging. Claudia Messerschmidt, Ingo Lieberwirth and Christoph Sieber are thanked for the cross-sectional TEM measurements and helpful discussions.

\section{Notes and references}

1 Z. Zhang and S. C. Glotzer, Nano Lett., 2004, 4, 1407-1413.

2 A. B. Pawar and I. Kretzschmar, Macromol. Rapid Commun., 2010, 31, 150-168.

3 J. Du and R. K. O'Reilly, Chem. Soc. Rev., 2011, 40, 2402-2416. 4 P. G. De Gennes, Rev. Mod. Phys., 1992, 64, 645-648.

5 A. Walther and A. H. E. Müller, Chem. Rev., 2013, 113, 51945261.

6 S. Y. Zhang, Z. Li, S. Samarajeeva, G. R. Sun, C. Yang and K. Wooley, J. Am. Chem. Soc., 2011, 133, 11046-11049.

7 J. A. Champion, Y. K. Katare and S. Mitragotri, Proc. Natl. Acad. Sci. U. S. A., 2007, 104, 1190111904.

8 R. Langer and D. A. Tirrell, Nature, 2004, 428, 487-492.

9 R. H. Staff, M. Gallei, M. Mazurowski, M. Rehahn, R. Berger, K. Landfester and D. Crespy, ACS Nano, 2012, 6, 9042-9049. 10 M. Grätzel, Nature, 2001, 414, 338-344.

11 T. Nisisako, T. Torii, T. Takahashi and Y. Takizawa, Adv. Mater., 2006, 18, 1152-1156.

12 C. M. Liddell, C. J. Summers and A. M. Gokhale, Mater. Charact., 2003, 50, 69-79.

13 O. Cayre, V. N. Paunov and O. D. Velev, J. Mater. Chem., 2003, 13, 2445-2450.

14 H. Takei and N. Shimizu, Langmuir, 1997, 13, 1865-1868.

15 G. A. Ozin, I. Manners, S. Fournier-Bidoz and A. Arsenault, Adv. Mater., 2005, 17, 3011-3018.
16 M. D. McConnell, M. J. Kraeutler, S. Yang and R. J. Composto, Nano Lett., 2010, 10, 603-609.

17 J. Q. Cui and I. Kretzschmar, Langmuir, 2006, 22, 8281-8284. 18 L. Hong, S. Jiang and S. Granick, Langmuir, 2006, 22, 94959499.

19 A. D. Dinsmore, M. F. Hsu, M. G. Nikolaides, M. Marquez, A. R. Bausch and D. A. Weitz, Science, 2002, 298, 1006-1009.

20 F. Yan and W. A. Goedel, Chem. Mater., 2004, 16, 1622-1626.

21 L. Wang, L. Xia, G. Li, S. Ravaine and X. S. Zhao, Angew. Chem., Int. Ed., 2008, 47, 4725-4728.

22 Z. K. Zhang, P. Pfeiderer, A. B. Schofield, C. Clasen and J. Vermant, J. Am. Chem. Soc., 2011, 133, 392-395.

23 A. B. Pawar and I. Kretzschmar, Langmuir, 2009, 25, 90579063.

24 A. B. Pawar and I. Kretzschmar, Langmuir, 2008, 24, 355-358. 25 Z. He and I. Kretzschmar, Langmuir, 2012, 28, 9915-9919.

26 C. E. Snyder, A. M. Yake, J. D. Feick and D. Velegol, Langmuir, 2005, 21, 4813-4815.

27 G. Zhang, D. Wang and H. Möhwald, Angew. Chem., Int. Ed., 2005, 44, 7767-7770.

28 G. Zhang, D. Wang and H. Möhwald, Nano Lett., 2005, 5, 143-146.

29 T. T. Chastek, S. D. Hudson and V. A. Hackley, Langmuir, 2008, 24, 13897-13903.

30 K. H. Roh, D. C. Martin and J. Lahann, J. Am. Chem. Soc., 2006, 128, 6796-6797.

31 K. H. Roh, D. C. Martin and J. Lahann, Nat. Mater., 2005, 4, 759-763.

32 Z. Nie, W. Li, M. Seo, S. Xu and E. Kumacheva, J. Am. Chem. Soc., 2006, 128, 9408-9412.

33 A. H. Gröschel, F. H. Schacher, H. Schmalz, O. V. Borisov, E. B. Zhulina, A. Walther and A. H. E. Müller, Nat. Commun., 2012, 3, 710.

34 Z. Li, E. Kesselman, Y. Talmon, M. A. Hillmyer and T. P. Lodge, Science, 2004, 306, 98-101.

35 R. Zheng, G. Liu and X. Yan, J. Am. Chem. Soc., 2005, 127, 15358-15359.

36 R. H. Staff, P. Rupper, I. Lieberwirth, K. Landfester and D. Crespy, Soft Matter, 2011, 7, 10219-10226.

37 Q. Chen, S. C. Bae and S. Granick, J. Am. Chem. Soc., 2012, 134, 11080-11083.

38 R. N. K. Taylor, H. Bao, C. Tian, S. Vasylyev and W. Peukert, Langmuir, 2010, 26, 13564-13571.

39 H. Bao, W. Peukert and R. N. K. Taylor, Adv. Mater., 2011, 23, 2644-2649.

40 H. Bao, B. Butz, Z. Zhou, E. Spiecker, M. Hartmann and R. N. K. Taylor, Langmuir, 2012, 28, 8971-8978.

41 M. Okubo, Y. Murakami and T. Fujiwara, Colloid Polym. Sci., 1996, 274, 520-524.

42 M. Okubo, T. Fujiwara and A. Yamaguchi, Colloid Polym. Sci., 1998, 276, 186-189.

43 M. Okubo, A. Yamaguchi and T. Fujiwara, Colloid Polym. Sci., 1999, 277, 1005-1008.

44 T. Fujibayashi, Y. Komatsu, N. Konishi, H. Yamori and M. Okubo, Ind. Eng. Chem. Res., 2008, 47, 6445-6449.

45 N. Konishi, T. Fujibayashi, T. Tanaka, H. Minami and M. Okubo, Polym. J., 2010, 42, 66-71. 
46 H. R. Sheu, M. S. El-Aasser and J. W. Vanderhoff, J. Polym. Sci., Part A: Polym. Chem., 1990, 28, 629-651.

47 J. W. Kim, R. J. Larsen and D. A. Weitz, J. Am. Chem. Soc., 2006, 128, 14374-14377.

48 J. W. Kim, R. J. Larsen and D. A. Weitz, Adv. Mater., 2007, 19, 2005-2009.

49 D. Crespy and K. Landfester, Beilstein J. Org. Chem., 2010, 6, 1132-1148.

50 R. H. Staff, D. Schaeffel, A. Turshatov, D. Donadio, H. J. Butt, K. Landfester, K. Koynov and D. Crespy, Small, 2013, DOI: 10.1002/smll.201300372.

51 D. Schaeffel, R. H. Staff, H. J. Butt, K. Landfester, D. Crespy and K. Koynov, Nano Lett., 2012, 12, 6012-6017.

52 Y. Zhao, K. Landfester and D. Crespy, Soft Matter, 2012, 8, 11687-11696.

53 Y. Zhao, J. Fickert, K. Landfester and D. Crespy, Small, 2012, 8, 2954-2958.
54 J. Fickert, C. Wohnhaas, A. Turshatov, K. Landfester and D. Crespy, Macromolecules, 2013, 46, 573-579.

55 J. Brandrup, E. H. Immergut and E. A. Grulke, Polymer Handbook, John Wiley and Sons, New York, 4th edn, 1999, p. VI/202.

56 G. Floudas, P. Placke, P. Stepanek, W. Brown, G. Fytas and K. L. Ngai, Macromolecules, 1995, 28, 6799-6807.

57 J. Brandrup, E. H. Immergut and E. A. Grulke, Polymer Handbook, John Wiley and Sons, New York, 4th edn, 1999, p. III/14.

58 D. W. van Krevelen and K. te Nijenhuis, Properties of Polymers, Elsevier Science: Amsterdam, 4th edn, 2009, p. 79.

59 J. S. Trent, J. I. Scheinbeim and P. R. Couchman, Macromolecules, 1983, 16, 589-598.

60 Z. K. Zhong and X. S. Sun, J. Appl. Polym. Sci., 2001, 81, 166-175.

61 M. Hermant, L. Klumperman, C. Koning and P. van der Schoot, WO 2009033933A2, 2009. 